\title{
Parois et vortex en micromagnétisme
}

\author{
Tristan Rivière
}

\begin{abstract}
Résumé
Nous présenterons l'énergie libre modélisant les états (polarisations) des materiaux ferromagnetiques. Le problème variationnel associé contient de nombreux régimes asymptotiques dans lesquels "on voit" se former des défauts du type vortex, du type paroi (Bloch et Neel Walls) ou du type mixte paroi-vortex (Cross-Tie Walls). Le but de cet exposé est de présenter les travaux qui s'efforcent de donner une justification mathématiqueà la création de ces singularités. Nous décrirons l'insuffisance des méthodes classiques de l'analyse fonctionnelle linéaire à rendre compte de ces phénomènes de perte de régularité et introduirons une approche mettant en oeuvre des outils de la théorie de la mesure géometrique appliqués a l'analyse des équations aux dérivees partielles. L'analyse des défauts en micromagnétisme a suscité des questions théoriques sur les lois de conservation scalaires non-linéaires. Nous presenterons à cette occasion un résultat récent géneralisant le phénomène de régularisation de Lax-Oleinick pour les lois scalaire à non-linearité strictement convexe $\frac{\partial u}{\partial t}+\frac{\partial f(u)}{\partial x}=0\left(f^{\prime \prime}>0\right)$, au cas où la distribution entropique de sauts n'est pas forcément de signe uniforme mais est une mesure signée quelconque : pour tout $S$ dans $C^{2}, \frac{\partial S(u)}{\partial t}+\frac{\partial \Phi(u)}{\partial x}$ est une mesure de Radon $\left((S, \Phi)\right.$ paire entropie flux : $\left.\Phi^{\prime}=S^{\prime} f^{\prime}\right)$. Nous démontrons alors que dans ce cas, en dimension $1+1$, pour une donnée initiale mesurable et bornée quelconque, les ondes de chocs sont contenues dans une union au plus dénombrable de courbes $C^{1}$.
\end{abstract}

\section{Des problèmes variationnels en physiques du micromagné- tisme.}

\section{1. l'énergie libre ferromagnétique.}

Les états d'un matériau ferromagnétique en l'abscence de champs extèrieur ou de courant extèrieur imposés sont régit par un lagrangien, l'énergie libre $G$, fonction de la polarisation magnétique $J$ (ou magnétisation) du matériau. $J$ s'écrit $J=J_{s} u$ où $u$ est un champ de vecteur unitaire sur $\Omega$, le domaine occupé par le matériau, et $J_{s}$ est le paramètre de saturation magnétique qui peut être supposé uniforme sur $\Omega$. 
Le flux magnétique $B$ s'écrit $B=\mu_{0} H+J$ où ( $\mu_{0}$ est la constante de perméabilité du vide) et $H$ le champs magnétique. En l'abscence de champ extérieur imposé, le champs magnétique est réduit au champs parasite $H_{d}$ (ou champ démagnétisant) donné par $H_{d}=\nabla \Phi_{d}$ où $\Phi_{d}$ est le potentiel solution de $\mu_{0} \Delta \Phi_{d}=-\operatorname{div} J$, si bien que l'on retrouve l'équation de Maxwell $\operatorname{div} B=0$. $-\operatorname{div} J$ agit alors comme une "charge magnétique" générant le potentiel $\Phi_{d}$. L'énergie libre ferromagnétique s'écrit alors

$$
G(u)=A \int_{\Omega}|\nabla u|^{2}+K \int_{\Omega} \Psi(u)+\frac{J_{s}^{2}}{2 \mu_{0}} \int_{\mathbb{R}^{3}}\left|H_{d}\right|^{2}
$$

Seule l'énergie de magnétostriction issue de la résistance élastique à des polarisations non uniformes est ici négligée.

$G$ est donc la somme de trois termes:

- L'énergie de rigidité d'échange, $A \int_{\Omega}|\nabla u|^{2}$ est un terme d'énergie élastique ordinaire qui tend à rendre uniforme la polarisation. Le coefficient d'échange $A \simeq[\mathrm{J} / \mathrm{m}]$ est un paramètre du matériau.

- L'énergie d'anisotropie $K \int_{\Omega} \Psi(u)$ tend a favoriser et pénaliser certaines directions de la polarisation $u$. Elle est très variable d'un matériau à l'autre . On distingue les anisotropies axiales ou multiaxiales (par exemple $\Psi_{\mathbf{u}}(u)=\left(1-u_{1}^{2}\right)$, axe $\vec{i}$ privilégié) des anisotropies planaires dites surfaciques intervenant principalement dans les cas de matériaux minces (ex. $\left.\Psi_{\mathbf{s}}(u)=u_{3}^{4}\right)$. Le coefficient d'anisotropie $K \simeq\left[\mathrm{J} / \mathrm{m}^{3}\right]$ est un paramètre du matériau mesurant l'intensité de l'anisotropie.

- L'énergie démagnétisante est la norme $L^{2}$ du champ démagnétisant $H_{d}$ multiplié par le coefficient $K_{d}=\frac{J_{s}^{2}}{2 \mu_{0}}$.

Le lecteur est invité à consulter l'excellent livre de A. Hubert et R. Schäfer [HS] pour une description plus complète de la modélisation mathématique du micromagnétisme.

\subsection{Le cas de la dimension 2 .}

Dans cet exposé nous étudions la fonctionnelle $G$ en dimension 2, pour un domaine $\Omega$ borné régulier et simplement connexe de $\mathbb{R}^{2}$ et en présence de l'anisotropie planaire $K \int_{\Omega} u_{3}^{4}=K \int_{\Omega}\left(1-u_{1}^{2}-u_{2}^{2}\right)^{2}$. Après renormalisation $\left(E:=G / K_{d}\right)$ nous avons le problème variationnel suivant :

$$
E(u)=d^{2} \int_{\Omega}|\nabla u|^{2}+Q \int_{\Omega}\left(1-u_{1}^{2}-u_{2}^{2}\right)^{2}+\int_{\mathbb{R}^{2}}|H|^{2}
$$

où $u$ est une application de $\Omega$ dans $S^{2}$ et $H$ est le champ de vecteur planaire suivant : Soit $\tilde{u}$ l'éxtension de $\left(u_{1}, u_{2}\right)$ par 0 en dehors de $\Omega$, et soit $\tilde{u}=-\nabla \Phi+\nabla^{\perp} g$ la décomposition de Hodges dans $L^{2}\left(R^{2}\right)$ de $\tilde{u}$ où $\nabla^{\perp} g$ est un rotationel en dimension 2 c'est à dire $\nabla^{\perp} g=\left(-\partial_{x_{2}} g, \partial_{x_{1}} g\right)$. Le champ démagnétisant $H$ est alors $H=\nabla \Phi$. La division de $G$ par le coefficient $K_{d}=\frac{J_{s}^{2}}{2 \mu_{0}}$ génère 2 nouveaux paramètre : le nouveau paramètre d'échage $d=\sqrt{A / K_{d}} \simeq[m]$ et le facteur de qualité qui caractérise, pour un matériau donné, la prédominance ou non de l'anisotropie sur l'effet démagnétisant du champ parasite $H$. 
La richesse et la complexité de ce problème variationnel est du à la combinaison de sa non-convexité ( par exemple issue de la contrainte ponctuelle $|u|=1$ ), de sa non-localité ( $H$ est issue d'une opération non-locale sur $u$ ) et de l'influence des divers paramètres $d, Q$ et $\Omega$ qui annoncent des régimes assymptotiques variés. La complexité est accrue dans le problème en 3 dimensions où, par exemple, l'épaisseur $l$ de $\Omega$ devient un paramètre supplémentaire. La limite $l$ tend vers 0 a un intéret physique particulier et correspond à l'étude des films minces micromagnétiques. Elle suscite des travaux mathématiques nombreux en particulier sous l'impulsion du groupe formé de A.DeSimone, R.Kohn, S. Müller et F. Otto (voir [DKMO2], [DKMO3]).

\section{Divers régimes assymptotiques.}

Dans la réalité physique le paramètre d'échange $d \simeq[m]$ est très petit devant la taille moyenne des échantillons férromagnétiques observés, $d<<\operatorname{diam}(\Omega)$. Par exemple pour le fer $d \simeq 10^{-9} \mathrm{~m}$, alors que la taille moyenne des échantillons est $\operatorname{diam} \Omega \simeq 10-100 \mu \mathrm{m})$. Il est donc naturel de se restreindre à la limite assymptotique $d \rightarrow 0$. On se propose alors de suivre des suites de polarisations $u_{d}$, lorsque $d$ tend vers 0 , qui ne sont ni forcément des états fondamentaux de $u$, ni même des points critiques, mais simplement des configurations telles que

$$
E_{d}\left(u_{d}\right)=O\left(\min E_{d}(u)\right) \quad \text { lorsque } \quad d \rightarrow 0
$$

Dans cette limite, l'effet uniformisant de l'energie d'échange sur la polarisation $u$ disparaît peu à peu et on s'attend à la formation de singularités. Elles peuvent être de plusieurs types et de différentes dimensions. On peur avoir des singularités de codimension 1 (parois), de codimension 2 (vortex) ou des combinaisons des deux (parois-vortex). Trois types particuliers de ces singularités apparaîssent souvent en physique du micromagnétisme et on reçu des appélations spécifiques.

(i) les parois de Néel : Elle apparaissent dans les cas de fortes anisotropies surfaciques et se caractérisent par des changements d'orientation brutales de la polarisation sur une zone de transition concentrée sur des hypersurfaces (en dimension 2 : des lignes). Le chemin adopté par la polarisation pour passer d'une orientation à l'autre de part et d'autre de la parroi (le profil) est contenu dans un plan et est localement invariant par translation le long de la paroi.

(ii) les parois de Bloch: A nouveau dans ce cas la zone de transition brutale se concentre le long d'hypersurfaces mais avec cette fois un profil qui peut beneficier des trois dimensions pour passer d'une polarisation à l'autre de part et d'autre de la paroi.

(iii) les parois en noeud de cravatte "cross tie walls" : Dans le cas de telles transitions on perd l'invariance par translation du profil le long de la paroi car viennent s'insérer, en des configurations périodiques, des vortex de codimension 2 autour desquelles la polarisation, à valeur dans un plan, réalise un degré non nul dans l'intersection de la sphère $S^{2}$ avec ce plan.

On distingue 2 types de matériaux :

- les materiaux magnétiques doux : ce sont ceux pour lesquels le facteur sans dimension $Q<<1$ (l'anisotropie est faible par rapport à l'effet du champ déma- 
gnétisant). Pour le fer $Q \simeq 10^{-2}$ et pour certains alliages fer-nickel on peut avoir jusqu'à $Q \simeq 10^{-4}$

- les matériaux magnétiques permanents : ce sont les matériaux à grand facteur de qualité $Q>>1$. Par exemple le samarium cobalt $\mathrm{SmCo}_{5}, Q \simeq 10^{2}$.

Dans la limite $d \rightarrow 0$ on extrait alors les problèmes mathématiques suivants :

Le prolème $\mathbf{A}_{0}$ : Dans le régime de très forte anisotropie planaire $Q>>1$ dans (1.2) une première approximation consiste à prendre directement $u_{3}=0$, si bien que $u$ est à valeur dans $S^{1}$. L'énergie s'écrit alors

$$
E^{A_{0}}(u)=d^{2} \int_{\Omega}|\nabla u|^{2}+\int_{\mathbb{R}^{2}}|H|^{2}
$$

Il n'y a désormais qu'un paramètre $d$ qui est petit et qui sera noté $\varepsilon$ par la suite. Dans [RS1], [RS2], [LR1]...sont étudiés le comportement des suites de polarisations $u_{d}$ satisfaisant (1.3). Ce modèle est simplificateur au sens suivant : toute application $u \in W^{1,2}\left(\Omega, S^{1}\right)$ admet un relèvement $\phi$ dans $W^{1,2}(\Omega, \mathbb{R})\left(u=e^{i \phi}\right)$. En effet pour un tel $u$ on démontre que $\operatorname{div}\left(u^{-1} \nabla^{\perp} u\right)=0$ et on choisit alors $\nabla \phi=u^{-1} \nabla u$. Une telle application ne peut alors avoir de degré sur une courbe fermée donnée de $\Omega$ et les vortex sont exclus. Dans ce problème on ne peut s'attendre qu'à observer des parois de Néel étudiées dans la section suivante.

Le problème A : Il s'agit toujours ici du régime fortement anisotropique, mais, afin de remédier à l'exclusion des vortex dans l'approximation précédente, on relache la contrainte $u_{3}=0$ et on choisit une dépendance de $Q$ en $d$ de la forme $Q=\frac{1}{d^{\alpha}}$ pour un nombre $\alpha>0$. Cela donne la fonctionnelle

$$
E^{A}(u)=d^{2} \int_{\Omega}|\nabla u|^{2}+\frac{1}{d^{\alpha}} \int_{\Omega} u_{3}^{4}+\int_{\mathbb{R}^{2}}|H|^{2} .
$$

Il est démontré dans [ARS] que les parois de Néel ne sont alors optimales que pour des sauts de la polarisation inférieurs à $\frac{\pi}{2}$ et que pour des sauts strictement plus grands les parois en noeud de cravatte sont énergétiquement plus favorables. Les configurations périodiques optimales des ces mélanges lignes de sauts-vortex y sont explicitement calculés. Ce résultat est venu apporté une confirmation mathématique à des observations expérimentales (voir [HS]).

Le problème $\mathbf{B}$ : Il s'agit cette fois de la modélisation des matériaux ferromagnétiques doux $Q<<1$. On étudie la limite assymptotique lorsque $d$ tend vers 0 sous la contrainte $d^{2}<<Q|\Omega|$ (ce qui recouvre bien la réalité des valeurs des paramètres donnés par les expèriences - voir plus haut). En première approximation, étant donnée la domination de l'effet démagnétisant pour $Q<<1$, on peut prendre directement $u=\nabla^{\perp} g$ (tout écart à ce que $H=0$ etant fortement pénalisé) et la fonctionnelle devient alors

$$
E^{B}(\nabla g)=d^{2} \int_{\Omega}\left|\nabla^{2} g\right|^{2}+Q \int_{\Omega}\left(1-|\nabla g|^{2}\right)^{2}
$$

où, modulo une renormalisation, seul reste un paramètre (petit) $\varepsilon=d / \sqrt{Q}$, il donne alors la taille caracteristique des zones de transitions. La fonctionnelle $E^{B}$ est une fonctionnelle de type Ginzburg-Landau en physique de la supraconductivité mais 
sous la contrainte que le paramètre d'ordre $u$ soit un gradient. Cela a pour conséquence, dans l'assymptotique $\varepsilon \rightarrow 0$, de transformer totalement la nature du problème originèllement elliptique (sans la contrainte $u=\nabla^{\perp} g$ ) en une situation hyperbolique comme nous l'expliquons dans la section suivante. Cette assymptotique a donné lieu à de nombreux travaux : tout d'abord ceux de P.Aviles et Y.Giga [AG1], [AG2] puis vinrent les contributions de A.Desimone, R.Kohn, S. Müller et F. Otto [DKMO1], de L. Ambrosio, C. Delellis et C. Mantegazza [ADM], de P.E.Jabin et B.Perthame $[J P 1] \ldots$

\section{La formation des parois de Néel.}

On se propose dans cette section d'étudier la $\Gamma$-limite du problème $A_{0}$. Le changement d'échelle suivant, pour la fonctionnelle $E^{A_{0}}$, est justifié a posteriori par le fait que la limites des énergies d'états fondamentaux est un réel non nul :

$$
E_{\varepsilon}(u)=\varepsilon \int_{\Omega}|\nabla u|^{2}+\frac{1}{\varepsilon} \int_{\mathbb{R}^{2}}|H|^{2}=\varepsilon \int_{\Omega}|\nabla \phi|^{2}+\frac{1}{\varepsilon} \int_{\mathbb{R}^{2}}|H|^{2},
$$

où $u=e^{i \phi}$ pour une fonction $\phi$ dans $W^{1,2}(\Omega, \mathbb{R})$ et

$$
H=-(2 \pi)^{-1} \nabla(\log |x| * \operatorname{div} \tilde{u})
$$

avec $\tilde{u}=u$ dans $\Omega$ et $\tilde{u}=0$ dans $\mathbb{R}^{2} \backslash \Omega$.

La majoration. C'est l'étape la plus constructive de la $\Gamma$-limite où, au moyen de fonction tests, on "évalue" l'énergie minimale. Nous avons dans ce cas.

Proposition 1 [RS1]

$$
\limsup _{\varepsilon \rightarrow 0} \min E_{\varepsilon}(u) \leq 2|\partial \Omega|
$$

La majoration $\min E_{\varepsilon}(u)=O(1)$ nous dit que, pour une suite de configurations minimales, $H$ converge vers 0 dans $L^{2}$. Si la contrainte $|u|=1$ est préservée à la limite, on s'attend à converger vers une solution de l'équation eikonale

$$
\left\{\begin{array}{l}
|\nabla g|=1 \quad \text { dans } \mathcal{D}^{\prime}\left(\mathbb{R}^{2}\right) \\
g=\text { cte } \quad \text { sur } \partial \Omega
\end{array}\right.
$$

La majoration (2.2) est obtenue en partant de la solution de viscosité de (2.3) donnée par $g_{\star}(x)=\operatorname{dist}(x ; \partial \Omega)$. On considère une fonction $\phi_{\star}$ telle que $e^{i \phi}=\nabla^{\perp} g_{\star}$ dont on régularise les singularités 1-dimensionnelles en insérant le long de ces lignes des profils points critiques de la version 1-dimensionnelle de $E$. L'inégalité (2.2) est en fait une égalité comme nous le voyons plus bas.

La minoration. L'inégalité de Cauchy-Schwarz donne pour toute configuration $u$ d'énergie finie

$$
E_{\varepsilon}(u)=\varepsilon \int_{\Omega}|\nabla \phi|^{2}+\frac{1}{\varepsilon} \int_{\mathbb{R}^{2}}|H|^{2} \geq 2 \int_{\Omega}|\nabla \phi \cdot H|
$$


On observe alors que pour tout $\phi$ dans $W^{1,2}(\Omega, \mathbb{R})$ et $H$ tel que $\operatorname{div}\left(H+e^{i \phi}\right)=0$, $\nabla \phi \cdot H$ admet l'écriture en divergence suivante

$$
\nabla \phi \cdot H=\operatorname{div}\left(\phi\left(e^{i \phi}+H\right)+e^{i\left(\phi+\frac{\pi}{2}\right)}\right)
$$

On considère une suite $\phi_{\varepsilon}$ de configurations satisfaisant (1.3) et convergeant fortement dans un $L^{p}(p>1)$ vers une limite $\phi$. La convergence forte permet de passer à la limite dans les non-linéarités et, au vue de la convergence de $H$ vers 0 dans $L^{2}$, on obtient, d'une part, que $\operatorname{div}\left(\phi\left(e^{i \phi}\right)+e^{i\left(\phi+\frac{\pi}{2}\right)}\right)$ est une mesure de Radon et, d'autre part, que

$$
\liminf _{\varepsilon \rightarrow 0} E_{\varepsilon}\left(e^{i \phi_{\varepsilon}}\right)=\int_{\Omega}\left|\operatorname{div}\left(\phi\left(e^{i \phi}\right)+e^{i\left(\phi+\frac{\pi}{2}\right)}\right)\right|
$$

La convergence forte donne aussi l'existence de $g$ satisfaisant (2.3) avec $g=0$ sur le bord de $\Omega$. On introduit les sous-domaines $\Omega_{ \pm}=g^{-1}\left(\mathbb{R}_{ \pm}\right) \cap \Omega$. Une intégration par partie formelle donne

$$
\begin{aligned}
& 2 \int_{\Omega}\left|\operatorname{div}\left(\phi\left(e^{i \phi}\right)+e^{i\left(\phi+\frac{\pi}{2}\right)}\right)\right| \geq 2 \sum_{ \pm}\left|\int_{\Omega_{ \pm}} \operatorname{div}\left(\phi\left(e^{i \phi}\right)+e^{i\left(\phi+\frac{\pi}{2}\right)}\right)\right| \\
& \quad \geq 2 \sum_{ \pm} \mathcal{H}^{1}\left(\partial \Omega_{ \pm}\right) \geq 2|\partial \Omega|
\end{aligned}
$$

Ce calcul peut être rendu rigoureux (voir [RS1]). De (2.6) on conclut immédiatement que, si la convergence forte s'étend à des suites arbitraires de configurations $\phi_{\varepsilon}$ satisfaisant (1.3), l'inégalité (2.2) est en fait une égalité et la minimisation du membre droit de (2.6) sur l'espace des $\phi$ tel que $\operatorname{div}\left(\phi\left(e^{i \phi}\right)+e^{i\left(\phi+\frac{\pi}{2}\right)}\right)$ soit une mesure de Radon devient un bon candidat pour la $\Gamma$ convergence.

La règle de Leibnitz sur les dérivations implique que la quantité $\operatorname{div}\left(\phi\left(e^{i \phi}\right)+\right.$ $\left.e^{i\left(\phi+\frac{\pi}{2}\right)}\right)$ s'annule pour toute fonction $\phi$ régulière satisfaisant $\operatorname{div}\left(e^{i \phi}\right)=0$. Lorsque $\phi$ n'est plus régulière la règle de Leibnitz peut être rompue. En particulier, pour une configuration $\phi$ égale à une constante $\phi_{ \pm}$de part et d'autre d'une droite $L$ de normale $\nu$ et satisfaisant la condition de Rankine-Hugoniot $e^{i \phi_{+}} \cdot \nu=e^{i \phi_{-}} \cdot \nu$ de façon à ce que dive $e^{i \phi}=0$ dans $\mathcal{D}^{\prime}\left(\mathbb{R}^{2}\right)$, on a

$$
\operatorname{div}\left(\phi\left(e^{i \phi}\right)+e^{i\left(\phi+\frac{\pi}{2}\right)}\right)=X \cos X-\sin X \mathcal{H}^{1}\llcorner L
$$

où $\mathcal{H}^{1}\left\llcorner L\right.$ est la restriction à $L$ de la mesure de Hausdorff 1 -dimensionnelle $\mathcal{H}^{1}$ et $X$ est le demi-saut $\frac{\phi_{+}-\phi_{-}}{2}$.

La compacité : Dans [RS1], [RS2] il est démontré que toute suite $\phi_{\varepsilon}$ bornée dans $L^{\infty}(\Omega)$, convergeant faiblement dans $L^{\infty, \star}$, et d'énergie $E_{\varepsilon}\left(e^{i \phi_{\varepsilon}}\right)$ uniformément bornée, converge fortement dans $L^{p} \forall p<+\infty$. Ce résultat est en fait une perturbation de la proposition suivante.

Proposition 2 Soit

$$
\mathcal{C}=\left\{\begin{array}{c}
\phi \in W^{1,2}\left(D^{2},[-\pi,+\pi]\right) \quad \text { tel que } \quad \operatorname{div}\left(e^{i \phi}\right)=0 \\
\text { et } \operatorname{div}\left(\phi\left(e^{i \phi}\right)+e^{i\left(\phi+\frac{\pi}{2}\right)}\right)=0
\end{array}\right\} .
$$

Etant donnée une suite $\phi_{n} \in \mathcal{C}$ convergeant au sens des distributions vers $\phi$, alors la convergence et forte dans $L^{p}(\forall p<+\infty)$ et $\phi$ est dans $\mathcal{C}$. 
Preuve: Il s'agit d'une application de la compacité par compensation de Luc Tartar [Ta]. On introduit en effet les fonctions $g_{n}$ et $h_{n}$ bornées dans $W^{1, \infty}$ telles que $e^{i \phi_{n}}=\nabla^{\perp} g_{n}$ et $\phi_{n} e^{i \phi_{n}}+e^{i\left(\phi_{n}+\frac{\pi}{2}\right)}=\nabla^{\perp} h_{n}$. Modulo extraction de sous suites, on peut toujours supposer que $g_{n}$ et $h_{n}$ convergent faiblement dans $W^{1, p}(\forall p<+\infty)$ et uniformément dans $C^{0}$ vers $g$ et $h$. Par définition on a

$$
\nabla^{\perp} g_{n} \cdot \nabla h_{n}=1
$$

La structure en jacobien du membre de gauche de cette identité permet de passer à la limite faible si bien que l'on a $\nabla^{\perp} g \cdot \nabla h=1$. On introduit la mesure de Young $\nu_{x}(t)$ associée à la convergence faible de $\phi_{n}$ dans $L^{\infty, \star}$ (i.e. $\delta_{t=\phi_{n}(x)} \otimes d x \rightarrow \nu_{x}(t) \otimes d x$ au sens des mesures de Radon). En supposant que $\int_{-\pi}^{\pi} \sin t d \nu_{x}(t)=0$ (ce qui est toujours possible modulo une rotation) l'identité $\nabla^{\perp} g \cdot \nabla h=1$ s'écrit au moyen de $\nu$

$$
\int_{-\pi}^{\pi} \cos t d \nu_{x}(t) \times \int_{-\pi}^{\pi} t \sin t+\cos t d \nu_{x}(t)=1=\left(\int_{-\pi}^{\pi} d \nu_{x}(t)\right)^{2} .
$$

Une étude de fonction élémentaire donne alors que $\nu_{x}(t)$ est la masse de Dirac en 0 ce qui implique la convergence forte.

L'introduction des formulations cinétiques par P.E. Jabin et B. Perthame dans l'étude du micromagnétisme (pour le problème B. Voir [JP1]) a, entre autres, apporté un éclairage à la compacité ci dessus qui de compensation compacité devient compacité en moyenne. Elle a aussi permis de proposer un meilleur candidat que $\int_{\Omega}\left|\operatorname{div}\left(\phi\left(e^{i \phi}\right)+e^{i\left(\phi+\frac{\pi}{2}\right)}\right)\right|$ pour la $\Gamma$-limite comme nous l'expliquons plus bas.

\section{Le paramètre cinétique $a$ :}

Pour tout $a \in \mathbb{R}$ on introduit la notation $a \wedge \phi:=\min (\phi, a)$. Il est alors aisé de vérifier que pour tout $\phi$ assez régulier (lipshitz par exemple) on a

$$
\forall a \in \mathbb{R} \quad \operatorname{div} e^{i \phi}=0 \quad \Longrightarrow \operatorname{div} e^{i \phi \wedge a}=0 \quad .
$$

Ceci cesse d'être vrai lorsque $\phi$ a des sauts et on vérifie que pour tout $\phi \in L^{\infty}$ solution $\operatorname{de} \operatorname{div}\left(e^{i \phi}\right)=0$ on a

$$
\int_{a \in \mathbb{R}} \operatorname{div} e^{i \phi \wedge a} d a=\operatorname{div}\left(\phi\left(e^{i \phi}\right)+e^{i\left(\phi+\frac{\pi}{2}\right)}\right)
$$

En particulier l'integrale en $a \operatorname{des} \operatorname{div}\left(e^{i \phi \wedge a}\right)$ redonne la mesure $X \cos X-\sin X \mathcal{H}^{1}\llcorner L$ dans le cas du saut le long d'une droite évoqué en (2.8). Dans [RS2], au moyen du paramètre de coupure $a$, nous améliorons la minoration (2.6) et nous donnons la formulation cinétique suivante au problème $A_{0}$.

Proposition 3 [RSQ] Soit $\phi_{\varepsilon}$ une suite de fonctions dans $W^{1,2}(\Omega, \mathbb{R})$ de norme $L^{\infty}$ et d'énergies $E_{\varepsilon}\left(e^{i \phi_{\varepsilon}}\right)$ uniformément bornées. On suppose par ailleurs que $\phi_{\varepsilon}$ converge au sens des distributions vers une limite $\phi_{\star}$. Alors, la convergence est forte dans tout $L^{p}(p<+\infty)$,

$$
\operatorname{div}\left(e^{i \phi_{\star} \wedge a}\right) \quad \text { est une mesure de Radon sur } \Omega \times \mathbb{R} \text {, }
$$


par ailleurs on a

$$
\liminf E_{\varepsilon}\left(e^{i \phi_{\varepsilon}}\right) \geq 2 \int_{a \in \mathbb{R}} \int_{\Omega}\left|\operatorname{div}\left(e^{i \phi_{\star} \wedge a}\right)\right| \geq 2|\partial \Omega|
$$

et $\phi_{\star}$ est solution de l'équation cinétique suivante

$$
i e^{i a} \cdot \nabla_{x}\left[\chi\left(\phi_{\star}(x)-a\right)\right]=\partial_{a}\left(\operatorname{div}\left(e^{i \phi_{\star} \wedge a}\right)\right) \quad \text { dans } \quad \mathcal{D}^{\prime}(\Omega \times \mathbb{R}),
$$

où $\chi(t)$ est la fonction caractéristique de $\mathbb{R}_{+}$.

Dans [RS2] nous donnons des exemples où la minoration (2.10) est meilleure que (2.6). Par ailleurs il est démontré dans [LR1] que pour tout $\phi \in L^{\infty}\left(D^{2}, \mathbb{R}\right)$ tel que $e^{i \phi}$ soit à divergence nulle, on a

$$
\phi \in \operatorname{Lip}(\Omega, \mathbb{R}) \quad \Longleftrightarrow \quad \operatorname{div}\left(e^{i \phi \wedge a}\right)=0 \quad \operatorname{dans} \mathcal{D}^{\prime}(\Omega \times \mathbb{R})
$$

(ce qui n'est pas le cas si on remplace $\operatorname{div}\left(e^{i \phi \wedge a}\right)=0$ dans $\mathcal{D}^{\prime}(\Omega \times \mathbb{R})$ par $\operatorname{div}\left(\phi e^{i \phi}+\right.$ $\left.e^{i\left(\phi+\frac{\pi}{2}\right)}\right)=0$ seulement).

\section{Le cas des suites minimisantes}

Un cas intérressant de la proposition 3 est celui des suites de configurations $\phi_{\varepsilon}$ telles que $\lim _{\varepsilon \rightarrow 0} E_{\varepsilon}\left(e^{i \phi_{\varepsilon}}\right)=\liminf _{\varepsilon \rightarrow 0} \min E_{\varepsilon}=2|\partial \Omega|$. Nous démontrons dans [RS2] que la limite $\phi_{\star}$ d'une telle suite vérifie la condition d'entropie

$$
\operatorname{div}\left(e^{i \phi_{\star} \wedge a}\right) \geq 0 \quad \operatorname{dans} \mathcal{D}^{\prime}(\Omega \times \mathbb{R})
$$

Cette positivité de la mesure de saut entropique dans le membre de droite de l'équation cinétique (2.11) rappelle des conditions de type Kruzhkov assurant l'unicité il est alors naturel d'espèrer montrer que tout solution entropique est la solution de viscosité donnée par $e^{i \phi_{\star}}=\nabla^{\perp} \operatorname{dist}(x, \partial \Omega$ ) (qui vérifie bien par ailleurs $\left.\int_{a} \int_{\Omega} \operatorname{div}\left(e^{i \phi_{\star} \wedge a}\right)=2|\partial \Omega|\right)$. L'approche "cinétique" de l'unicité (voir [Pe]) semble malheureusement ne pas s'adapter à la situation présente d'une équation cinétique (2.11) sans paramètre de temps. Le paramètre de temps est en fait caché dans l'équation (2.11) : il s'agit de la fonction $g_{\star}$ vérifiant $\nabla^{\perp} g_{\star}=e^{i \phi_{\star}}$. Par exemple, dans le cas très particulier où $\operatorname{div}\left(e^{i \phi_{\star} \wedge a}\right)=0$, en introduisant $h_{\star}$ telle que $\nabla^{\perp} h_{\star}=\phi\left(e^{i \phi}\right)+e^{i\left(\phi+\frac{\pi}{2}\right)}$, il est démontré dans [LR1] que $\phi_{\star}$ est solution de l'équation de Burger

$$
\frac{\partial \phi_{\star}}{\partial g_{\star}}+\frac{\partial \phi_{\star}^{2}}{\partial h_{\star}}=0
$$

Le problème de trouver une variable $h_{\star}$ "duale" de $g_{\star}$ dans le cas général afin d'exploiter l'équation cinétique pour l'unicité reste ouvert. C'est en utilisant des méthodes types "solutions de viscosité" que le problème de l'unicité a été résolu.

Theorem 2.1 [ALR] Soit $\phi_{\star}$ une fonction dans $L^{\infty}(\Omega, \mathbb{R})$ vérifiant div $\tilde{u}=0$, où $\tilde{u}$ est l'extension de $e^{i \phi_{\star}}$ par 0 en dehors de $\Omega$, on a

$$
\operatorname{div}\left(e^{i \phi_{\star} \wedge a}\right) \geq 0 \quad \text { dans } \mathcal{D}^{\prime}(\Omega \times \mathbb{R}) \quad \Longleftrightarrow \quad e^{i \phi_{\star}}=\nabla^{\perp} \operatorname{dist}(x, \partial \Omega) .
$$


La $\Gamma$-limite L'étude du problème $A_{0}$ nous amène naturellement à introduire l'espace

$$
\mathcal{M}_{\text {div }}(\Omega)=\left\{\begin{array}{ccc}
\phi \in L^{\infty}(\Omega, \mathbb{R}) & \text { telle que } & \operatorname{div}\left(e^{i \phi}\right)=0 \\
\text { et } \operatorname{div}\left(e^{i \phi \wedge a}\right) & \text { est une mesure de Radon sur } \Omega
\end{array}\right\} .
$$

L'ensemble des résultats ci-dessus fait de la fonctionnelle $\int_{a} \int_{\Omega}\left|\operatorname{div}\left(e^{i \phi \wedge a}\right)\right|$ sur l'espace $\mathcal{M}_{\text {div }}(\Omega)$ un candidat très plausible pour la $\Gamma$-limite du problème $A_{0}$. Afin de vérifier que c'est bien le cas il reste à démontrer le fait suivant : étant donné un élement $\phi$ de $\mathcal{M}_{\text {div }}(\Omega)$, il existe une suite $\phi_{\varepsilon}$ dans $W^{1,2}(\Omega, \mathbb{R}) \cap L^{\infty}$ dont l'énergie $E_{\varepsilon}\left(e^{i \phi_{\varepsilon}}\right)$ ainsi que la norme $L^{\infty}$ restent uniformément bornées et convergeant vers $\phi$. L'approche constructive semble être ici la plus adaptée : il s'agirait alors d' "itentifier" l'ensemble singulier de $\phi$ "portant" la mesure $\operatorname{div}\left(e^{i \phi \wedge a}\right)$ et de le résorber en insérant des "profils" à l'echelle $\varepsilon$. Toute tentative de ce genre, en vue d'étabir la $\Gamma$ - limite, requiert une connaissance approfondie de l'espace $\mathcal{M}_{\text {div }}(\Omega)$ et de la nature des singularités d'un élément quelconque $\phi$ de $\mathcal{M}_{\text {div }}(\Omega)$.

Dans un premier temps on cherche à comparer notre espace non linéaire $\mathcal{M}_{\text {div }}(\Omega)$ avec les espaces linéaires fonctionnels existants. Il s'agit de trouver des espaces linéaires $L(\Omega)$ aussi petits possible tels que $\mathcal{M}_{\text {div }}(\Omega) \subset L(\Omega)$. A-t-on par exemple $\mathcal{M}_{\text {div }}(\Omega) \subset B V(\Omega)$ ? et peut-on parler alors de l'ensemble des sauts d'un élément arbitraire $\phi \in \mathcal{M}_{\text {div }}(\Omega)$ ?

Cet espoir est en fait trop optimiste, et L.Ambrosio, C. Dellelis et C. Mantegazza montrent le résultat suivant :

Theorem 2.2 [ADM]

$$
\mathcal{M}_{\text {div }}(\Omega) \propto B V(\Omega)
$$

Un élément $\phi$ dans $\mathcal{M}_{\text {div }}(\Omega)$ qui ne soit pas dans $B V(\Omega)$ est obtenu de la façon suivante : $\phi$ va être constante de part et d'autre d'un nombre dénombrable de ségments $S^{k}$ qui viennent s'accumuler sur l'axe horizontal. La condition de RankineHugoniot est préservée le long de chaque ségment $S^{k}$ ( c'est à dire $e^{i \phi_{+}^{k}} \cdot \nu^{k}=e^{i \phi_{-}^{k}} \cdot \nu$ où $\nu^{k}$ est la normale à $S^{k}$ ). Enfin, les angles $\phi_{ \pm}^{k}$ sont choisis de façon à ce que

$$
\sum_{k=1}^{\infty} \int_{S^{k}}\left|\phi_{+}^{k}-\phi_{-}^{k}\right|^{3}<+\infty \quad \text { et } \quad \sum_{k=1}^{\infty} \int_{S^{k}}\left|\phi_{+}^{k}-\phi_{-}^{k}\right|=+\infty
$$

La deuxième identité assure que $\phi$ n'est pas dans $B V$, alors que la première, au vue de la formule (2.8), en observant que $|X \cos X-\sin X|$ se comporte comme $X^{3}$ pour des petits angles, assure que $\int_{a} \int_{\Omega}\left|\operatorname{div}\left(e^{i \phi \wedge a}\right)\right|<+\infty$ et donc que $\phi \in \mathcal{M}_{\text {div }}$.

La formulation cinétique (2.11) peut-être mise à profit au moyen des techniques de régularité en moyenne afin d'obtenir de la régularité pour tout $\phi$ dans $\mathcal{M}_{\text {div }}$. En appliquant directement les résultats de R. DiPerna, P.L. Lions et Y. Meyer [DLM] il vient $\mathcal{M}_{\text {div }}(\Omega) \subset W^{\sigma, p}(\Omega)$ pour tout $\sigma<\frac{1}{5}$ et $p<\frac{5}{3}$. En prenant en compte la specificité de l'équation (2.11) P.E. Jabin et B. Perthame dans [JP2] améliorent ce résultat en montrant le théorème suivant.

Theorem 2.3 [JPQ]

$$
\forall \sigma<1 / 3 \quad \forall p<3 / 2 \quad \mathcal{M}_{d i v}(\Omega) \subset W^{\sigma, p}(\Omega) \quad .
$$


Ce théorème tout en étant un bel accomplissement des techniques d'analyse fonctionelle est cependant loin de nous informer sur la structure particulière des singularités d'éléments de $\mathcal{M}_{\text {div }}(\Omega)$ et l'éventuelle notion de sauts 1-dimensionnels pour un $\phi$ arbitraire dans $\mathcal{M}_{\text {div }}(\Omega)$ ( qui correspondrait plutôt à $W^{\sigma, p}$ pour $\sigma p \simeq 1$ ).

Nous abandonnons alors les techniques d'analyses fonctionnelles classiques pour étudier l'ensemble singulier d'un élément de $\mathcal{M}_{\text {div }}(\Omega)$ quelconque, directement au moyen d'outils de la théorie de la mesure géométrique.

Un théorème de structure pour $\mathcal{M}_{\text {div }}(\Omega)$.

Nous avons pour modèle de théorème de structure le cas classique de $B V(\Omega)$ (voir par exemple [AFP]).

Theorem 2.4 Soit $\phi \in B V(\Omega)$, alors il existe un sous ensemble $J_{\phi} \subset \Omega$ rectifiable (contenu dans une union au plus dénombrable de courbes $C^{1}$ ) telle que pour $\mathcal{H}^{1}$ presque tout point $x_{0}$ de $J_{\phi}$ où le vecteur tangent à $J_{\phi}$ existe, $\phi$ admet une limite approximative $\phi_{ \pm}\left(x_{0}\right)$ à gauche et à droite de la tangente. Par ailleurs $\phi$ est approximativement continue partout dans $\Omega \backslash J_{\phi}$ en dehors d'un ensemble de mesure $\mathcal{H}^{1}$ nulle. Enfin la dérivée distributionnelle de $\phi, D \phi$ se décompose en trois mesures perpendiculaires les unes aux autres:

$$
D \phi=\nabla \phi \mathcal{L}^{2}+D^{c} \phi+\left(\phi_{+}-\phi_{-}\right) \otimes \nu_{\phi} \mathcal{H}^{1}\left\llcorner J_{\phi}\right.
$$

où $\nabla \phi$ est une fonction de $L^{1}(\Omega)$ (la dérivée ponctuelle), $\nu_{\phi}$ est le vecteur normal à $J_{\phi}$ et $D^{c} \phi$ (la partie Cantor) est une mesure vérifiant

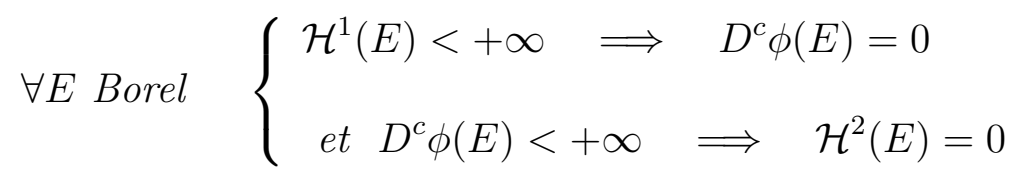

Dans [AKLR] il est établi indépendament de la théorie $B V(\Omega)$, inappropriée dans ce cas, le théorème de structure suivant :

Theorem 2.5 Soit $\phi \in \mathcal{M}_{\text {div }}(\Omega)$, alors il existe un sous ensemble $J_{\phi} \subset \Omega$ rectifiable (contenu dans une union au plus dénombrable de courbes $C^{1}$ ) tel que pour $\mathcal{H}^{1}$ presque tout point $x_{0}$ de $J_{\phi}$ où le vecteur tangent $\grave{a} J_{\phi}$ existe, $\phi$ admet une limite approximative $\phi_{ \pm}\left(x_{0}\right)$ à gauche et à droite de la tangente. Par ailleurs

$$
\mathcal{H}^{1} \text { p.p. } x_{0} \in \Omega \backslash J_{\phi} \quad \lim _{r \rightarrow 0} \frac{1}{\left|B_{r}\left(x_{0}\right)\right|} \int_{B_{r}\left(x_{0}\right)}|\phi-\bar{\phi}|=0
$$

où $\bar{\phi}=\frac{1}{\left|B_{r}\left(x_{0}\right)\right|} \int_{B_{r}\left(x_{0}\right)} \phi$. On a d'autre part

$$
\mu=\int_{a \in \mathbb{R}}\left|\operatorname{div}\left(e^{i \phi \wedge a}\right)\right|=|X \cos X-\sin X| \mathcal{H}^{1}\left\llcorner J_{\phi}+\delta_{\phi}\right.
$$

où $X=\frac{\phi_{+}-\phi_{-}}{2}$ et $\delta_{\phi}$ est une mesure perpendiculaire à $\mathcal{H}^{1}$ (la partie Cantor de $\mu$ ) :

$$
\forall E \text { Borel t.q. } \mathcal{H}^{1}(E)<+\infty \quad \Longrightarrow \quad \delta_{\phi}(E)=0
$$


Enfin on a pour presque tout $a \in \mathbb{R}$

$$
\operatorname{div}\left(e^{i \phi \wedge a}\right)\left\llcorner J_{\phi}=\chi_{\left\{\phi_{-}<a<\phi_{+}\right\}}\left(e^{i a}-e^{i \phi_{-}}\right) \cdot \nu_{\phi} \mathcal{H}^{1}\left\llcorner J_{\phi}\right.\right.
$$

où $\nu_{\phi}$ est le vecteur unitaire normal $\grave{a} J_{\phi}$ et $\chi_{\left\{\phi_{-}<a<\phi_{+}\right\}}$la fonction caractéristique de l'intervalle $\left(\phi_{-}, \phi_{+}\right)$.

L'ensemble rectifiable $J_{\phi}$ est la réunion des parois de Néel. Il est vraissemblable que la partie Cantor de la mesure $\mu$ soit toujours nulle. Si par exemple $J_{\phi}$ est contenu dans un ensemble fermé de mesure $\mathcal{H}^{1}$ finie, il est démontré dans [AKLR] que $\delta_{\phi}$ est nulle. Afin de démontrer que $\delta_{\phi}=0$ dans le cas général il suffirait de remplacée la condition de type VMO (2.18) obtenue par une condition d'approximative continuité, c'est à dire avoir (2.18) où $\bar{\phi}$ est remplacé par une constante indépendante de $r$. Ce problème est toujours ouvert. Sa résolution permettrait de terminer la $\Gamma$-limite pour le problème $A_{0}$.

Une des clefs de la preuve du théorème 2.5 est de démontrer que pour $\mu$ presque tout point $x_{0}$, pour toute suite arbitraire de dilatations de $\phi: \phi_{r_{i}}(x):=\phi\left(r_{i} x+x_{0}\right)$ avec $r_{i} \rightarrow 0$, modulo extraction d'une sous suite, on converge fortement vers une limite $\phi_{\infty}$ dont la mesure de saut s'écrit

$$
\operatorname{div}\left(e^{i \phi_{\infty} \wedge a}\right)=h(a) \lambda(x) \quad \operatorname{dans} \mathcal{D}^{\prime}\left(\mathbb{R}^{2} \times \mathbb{R}\right)
$$

pour une mesure $\lambda(x)$ positive et une fonction lipshitz $h$. On démontre ensuite que $\lambda(x)$ est indépendant de la suite $r_{i}$ choisie et que $\lambda(x)$ est une mesure uniforme portée par une droite qui est la tangente à $J_{\phi}$ dans le cas où $\lambda$ n'est pas identiquement nulle.

\section{La rectifiabilité des ondes de chocs pour des lois scalaires hyperboliques non-linéaires.}

P.D. Lax et O. Oleinick dans les années 50 méttaient en évidence un phénomène de régularisation tout à fait spectaculaire pour les solutions entropiques d'équations hyperboliques scalaires en dimension 1 d'espace et à non linéarité strictement convexe. Ils démontrent que pour de telles solutions issues d'une donnée initiale mesurable et bornée arbitraire deviennent spontanément $B V$ pour tout temps strictement positif et que donc, les ondes de chocs portant la mesure de saut entropique sont rectifiables. Pour une non-linéarité $f(t)$ strictement convexe et 2 réels arbitraires $a$ et $b$ on introduit la notation suivante :

$$
\Delta(a, b):=\frac{(a-b)^{2}\left[\frac{f(a)+f(b)}{2}\right]-(a-b) \int_{b}^{a} f(t) d t}{\left[(a-b)^{2}+(f(a)-f(b))^{2}\right]^{\frac{1}{2}}}
$$

(Il est important de retenir que cette quantité qui correspond, moyennant un coefficient borné, à la différence entre l'aire du trapèze $(a, 0),(b, 0),(b, f(b))$ et $(a, f(a))$ et l'aire sous le graphe de $f$ entre $a$ et $b$, se comporte en $\left.(a-b)^{3}\right)$. Le résultat de Lax-Oleinick s'énnonce alors ainsi. 
Theorem 3.1 [La][Ol] Soit $f$ une fonction $C^{2}$ strictement convexe. Soit $\phi_{0}(x) \in$ $L^{\infty} \cap L^{1}(\mathbb{R})$ et $\phi(x, t)$ solution de

$$
\left\{\begin{array}{l}
\frac{\partial \phi}{\partial t}+\frac{\partial}{\partial x}[f(\phi)]=0 \quad \text { dans } \mathcal{D}^{\prime}\left(\mathbb{R}_{+} \times \mathbb{R}\right) \\
\phi(0, x)=\phi_{0}(x)
\end{array}\right.
$$

telle que pour tout $a \in \mathbb{R}$ la condition entropique suivante soit vérifiée

$$
m(x, t, a) a=\frac{\partial}{\partial t}(\phi \wedge a)+\frac{\partial}{\partial x}[f(\phi \wedge a)] \geq 0
$$

alors $\phi$ est dans $B V_{\text {loc }}\left(\mathbb{R}_{+}^{*} \times \mathbb{R}\right)$ et il existe un sous ensemble $J_{\phi}$ de $\mathbb{R}_{+} \times \mathbb{R} 1$ dimensionnel rectifiable tel que

$$
\mu(x, t)=\int_{a \in \mathbb{R}}|m(x, t, a)| d a=\Delta\left(\phi_{+}, \phi_{-}\right) \mathcal{H}^{1}\left\llcorner J_{\phi}\right.
$$

L'approche adoptée plus haut pour mettre en évidence les parois de Néel s'adapte parfaitement à la situation présente afin d'étendre le phénomène de régularisation type Lax-Oleinick dans le cas général de distributions de sauts qui sont des mesures signées quelconque. Précisemment nous avons.

Theorem 3.2 [LR2] Soit $f$ une fonction $C^{2}$ strictement convexe. Soit $\phi_{0}(x) \in$ $L^{\infty} \cap L^{1}(\mathbb{R})$ et $\phi(x, t)$ solution de

$$
\left\{\begin{array}{l}
\frac{\partial \phi}{\partial t}+\frac{\partial}{\partial x}[f(\phi)]=0 \quad \text { dans } \mathcal{D}^{\prime}\left(\mathbb{R}_{+} \times \mathbb{R}\right) \\
\phi(0, x)=\phi_{0}(x)
\end{array}\right.
$$

telle que

$$
m(x, t, a)=\frac{\partial}{\partial t}(\phi \wedge a)+\frac{\partial}{\partial x}[f(\phi \wedge a)] \in \mathcal{M}_{l o c}\left(\mathbb{R} \times \mathbb{R}_{+}^{*} \times \mathbb{R}\right),
$$

où $\mathcal{M}$ désigne les mesures de Radon. Alors il existe un sous ensemble $J_{\phi}$ de $\mathbb{R}_{+} \times \mathbb{R}$ 1 dimensionnel rectifiable tel que

$$
\mu(x, t)=\int_{a \in \mathbb{R}}|m(x, t, a)| d a=\Delta\left(\phi_{+}, \phi_{-}\right) \mathcal{H}^{1}\left\llcorner J_{\phi}+\delta_{\phi}\right.
$$

où $\delta_{\phi}$ est la partie "Cantor" de $\mu$ satisfaisant

$$
\forall B \text { Borel } \quad \mathcal{H}^{1}(B)<+\infty \Longrightarrow \delta_{\phi}(B)=0
$$

Dans ce cas, à nouveau, l'étape clef consiste à montrer que les "blow-up" de solutions de l'équation (3.2) satisfont

$$
\frac{\partial}{\partial t}\left(\phi_{\infty} \wedge a\right)+\frac{\partial}{\partial x}\left[f\left(\phi_{\infty} \wedge a\right)\right]=h(a) \lambda(x, t)
$$


où $h$ est une fonction lipshitz puis on démontre que de telles solutions sont en fait $B V$. De façon générale il est légitime d'éspérer de la régularité $B V$ pour les solutions "blow-ups" de lois de conservation scalaires en dimension $n+1$, c'est à dire satisfaisant

$$
\frac{\partial \phi}{\partial t}+\sum_{j=1}^{n} \frac{\partial\left(f_{j}(\phi)\right)}{\partial x_{j}}=0
$$

et pour lesquelles la distribution de saut est une mesure de Radon qui s'écrit

$$
\frac{\partial}{\partial t}(\phi \wedge a)+\sum_{j=1}^{n} \frac{\left.\partial\left(f_{j}(\phi \wedge a)\right)\right)}{\partial x_{j}}=h(a) \lambda(x)
$$

où $h$ est une fonction lipshitz.

\section{Conclusion}

i) L'étude des problèmes du micromagnétismes montrent que les solutions "physiques" des lois de conservations scalaires ne sont pas forcement toujours les solutions entropiques mais plus généralement celle dont les distributions de sauts sont des mesures de Radon de signes quelconques.

ii) Il est raisonnable d'espérer étendre le theorème 3.2 aux solutions d'équations de (3.6) en dimension $n+1$ quelconque dans les cas où les conditions garantissant la compacité des suites de solutions de distributions de sauts uniformément bornée dans les mesures de Radon (voir un exemple de telles conditions dans [LPT]). Il est important de noter que dans tous ces cas $\Delta\left(\phi_{+}, \phi_{-}\right)$se comporte en $O\left(\left(\phi_{+}-\phi_{-}\right)^{3}\right)$ ce qui fait que la régularité $B V$ est à écarter. La rectifiabilité des sauts doit être obtenue indépendament de la théorie $B V$ en suivant les approches de [AKLR] et [LR2].

iii) Cet exposé présentes des approches alternatives à l'utilisation systématique de l'analyse fonctionnelle linéaire afin d'étudier la régularité de solutions d'équations aux dérivées partielles non-linéaires. Cette démarche qui consiste à travailler directement sur l'ensemble singulier afin d'établir une éventuelle structure géométrique particulière de cet ensemble et ensuite d'étendre cette régularité de l'ensemble singulier à celle de la solution elle même a été très fructueuse dans l'analyse d'équations elliptiques non-linéaires ( voir les travaux de Leon Simon et Fanghua Lin sur les applications harmoniques entre variétes et les surfaces minimales).

iv) L'idée d'introduire les outils de la théorie de la mesure géométrique dans l'analyse des lois de conservation hyperboliques non-linéaires a son origine dans les travaux de R. DiPerna et aussi C.M. Dafermos (voir par exemple [Di] et [Da]) où néanmoins seules des solutions $B V$ sont considérées. Notre effort plus haut a été de s'abstraire de cette hypothèse.

Au moment de la rédaction de cet exposé nous avons appris que Camillo Dellis et Felix Otto établissent dans un travail en cours un résultat de structure correspondant au théorème 2.5 pour le problème $B$. 


\section{Références}

[ARS] F. Alouges, T. Rivière and S. Serfaty, "Néel Walls and Cross-tie Walls for micromagnetic materials having a strong planar anisotropy", COCV (2002), Volume à la mémoire de J.L.Lions.

[ADM] L. Ambrosio, C. De Lellis, and C. Mantegazza, "Line energies for gradient vector fields in the plane", Calc. Var. PDE 9 (1999) 4, 327-355.

[AFP] L. Ambrosio, N. Fusco, and D. Pallara, "Functions of Bounded Variation and Free Discontinuity Problems", Oxford University Press, (2000).

[AKLR] L. Ambrosio, B. Kirchheim, M. Lecumberry and T. Rivière "Rectifiability of defect measures arising in micromagnetic domains" Volume dedicated to the 80th birthday of O.Ladyzhenskaya, Kluwer Academic (2002).

[ALR] L. Ambrosio, M. Lecumberry and T. Riviere, A viscosity property of minimizing micromagnetic configurations, submitted, (2002).

[AG1] P. Aviles and Y. Giga, A mathematical problem related to the physical theory of liquid crystals configurations, Proc. Centre Math. Anal. Austral. Nat. Univ., 12, (1987), 1-16.

[AG2] P. Aviles and Y. Giga, On lower semicontinuity of a defect obtained by a singular limit of the Ginzburg-Landau type energy for gradient fields, Proc. Royal Soc. Edinburgh, Sec A, 129, (1999), 1-17.

[Da] C.M. Dafermos "Generalized characteristics and the structure of solutions of hyperbolic conservation laws" Indiana University Math. J. 26 (1977) 1097-1119.

[DKMO1] A. DeSimone, R.V. Kohn, S. Müller and F. Otto, A compactness result in the gradient theory of phase transitions, to appear in Proc. Royal Soc. Edinburgh.

[DKMO2] A. DeSimone, R.V. Kohn, S. Müller and F. Otto, "Magnetic microstructures, a paradigm of multiscale problems", to appear in Proceedings of ICIAM, (1999).

[DKMO3] A DeSimone, R.V. Kohn, S. Müller and F. Otto, "A reduced theory for thin-film micromagnetics" to appear in CPAM (2002).

[Di] R.J. DiPerna "The structure of solutions to hyperbolic conservation laws" Non linear analysis and mechanics: Heriot-Watt Symposium, Vol IV Res. Notes in Math.39 Pitman, Boston (1979) 1-16.

[DLM] R. DiPerna, P.L. Lions and Y. Meyer " $L^{p}$ regularity of velocity averages" Annales IHP, Analyse non linéaire, 8, (1991), 271-287.

[HS] A. Hubert and R. Schäfer "Magnetic domains; the analysis of Magnetic Microstructures" Springer (1998).

[JP1] P.E. Jabin and B. Perthame, "Compactness in Ginzburg-Landau energy by kinetic averaging" Comm. Pure Appl. Math., 54, (2001), no 9, 1096-1109.

[JP2] P.E. Jabin and B. Perthame, "Regularity in kinetic formulations via averaging lemmas" prépublication (2002).

[La] P.D. Lax "Hyperbolic systems of conservation laws II" Comm. Pure Appl. Math. 10 (1957) 537-566. 
[LR1] M. Lecumberry and T. Rivière, "Regularity for micromagnetic configurations having zero jump energy", to appear in Calc. of Var. and P.D.E. (2002).

[LR2] M. Lecumberry and T. Rivière, "Rectifiability of Shock waves for some nonlinear hyperbolic conservation laws" prépublication (2002).

[LPT] P.L. Lions, B. Perthame and E. Tadmor "A kinetic formulation of multidimensional scalar conservation laws and related equations" J. A.M.S., 7(1994), no7, 169-191.

[Ol] O. Oleinik, "The cauchy problem for non-linear equations in a class of discontinuous functions" Dokl. Akad. Nauk SSSR 95 (1954), 451-454. English transl : AMS Trans. Ser.2 42, AMS, Providence, RI, (1964), 7-12.

[Pe] B. Perthame "Uniqueness and error estimates in first order quasilinear conservation laws via the kinetic entropy defect measure" J. Math. Pures Appl. 77, (1998), 1055-1064.

[RS1] T. Rivière and S. Serfaty, "Limiting Domain Wall Energy for a Problem Related to Micromagnetics", Comm. Pure Appl. Math., 54, (2001), 294-338.

[RS2] T. Rivière and S. Serfaty, "Compactness, kinetic formulation and entropies for a problem related to micromagnetics", to appear in Comm. P.D.E. (2002).

[Ta] L. Tartar "Compensated compactness and applications to partial differential equations" Non linear analysis and mechanics : Heriot-Watt Symposium, Vol IV Res. Notes in Math.39 Pitman, Boston (1979) 136-212. 